\title{
Testing Bell inequalities for multi-partite systems with frequency-encoded photonic qubits
}

\author{
V. O. Sheremetev, A. S. Rudenko, A. I. Trifanov \\ ITMO University, Kronverkskiy, 49, St. Petersburg, 197101, Russia \\ vlad.sheremetiev@gmail.com,plane2393@gmail.com, alextrifanov@gmail.com
}

DOI 10.17586/2220-8054-2018-9-4-484-490

\begin{abstract}
Generalizing the problem of state nonlocality measurement, we suggest a multi-partite Bell test for multi-photon frequency-entangled quantum state in a quantum network. Each side of this network is equipped with a generalized detector, consisting of an electro-optic phase modulator, frequency filter and photo-counter. In our paper, we develop a theory of Bell nonlocality measurement in frequency domain, using generalized Svetlichny inequalities. Solving the optimization problem for detectors inputs, we obtain optimal measurement parameters which allow strong violation of considered inequalities. As a particular case, we consider bi- and tripartite cases for EPR, GHZ and Wigner states correspondingly.
\end{abstract}

Keywords: Quantum nano-technologies, Bell nonlocality, spectral-entangled photons, phase modulation.

Received: 12 July 2018

\section{Introduction}

One of the most crucial experiment testing the validity of quantum mechanics is examination of nonlocal nature of quantum correlations [1]. Quantum entanglement [2], which is usually used as a basic resource for communication and information processing appears to be an echo of this quantum phenomenon [3-5]. In 1964, Bell [6] approved his famous theorem which states incompatibility between predictions of quantum theory and any physical theory satisfying notion of locality and hidden variable assumption. The Bell parameter $S$ being the function of detector's local settings and state of monitored composite system violates certain inequality in the case of nonlocal correlation existence. For the bipartite system, this may take the form of Clauser-Horne-ShimonyHolt [8] (CHSH) inequality $\left|S^{(2)}\right| \leq 2$, whereas in the tripartite case, we have Mermin $\left|S^{(3)}\right| \leq 2$ and Svetlicnhiy $\left|S^{(3)}\right| \leq 4$ counterparts $[9,10]$.

From the perspective of application in quantum nano-technologies and quantum communication protocols [11] it is very attractive to realize Bell test on photonic quantum states, which are widely used in quantum cryptography and information processing $[12,13]$. Current experimental methods in quantum optics allow generation of entanglement in many-photon systems as soon as manipulating by single- and multi-photon states in integral silica devices $[14,15]$. With these techniques, Bell inequalities were tested for bipartite and tripartite systems in the case of polarization and time-bin coding $[16,17]$. Frequency-bin coding is another attractive way for representation of quantum information units due to relatively simple technique of manipulating collective state of frequency-encoded qubits by phase shifters and electro-optical modulators $[18,19]$. By analogy, with linear optical quantum computation (LOQC), this branch of quantum information science was titled Spectral-LOQC (S-LOQC) [20]. Recently [21] it was reported about testing CHSH inequality in frequency domain for biphotons generated in spontaneous-downconversion process by choosing optimal parameters of modulation. In [22], the Bell inequality was tested on frequency-bin entangled photon pairs using time-resolved detection.

Here, we deal with generalization of the problem solved for bipartite case in [21,23]. In our model, $N$ communicating parts of quantum network realize Bell test on common multi-photon state. Each part of the network is equipped with a phase modulator, filter and photo-detector and receive only one photon. We estimate modulation parameters, which allow us to register nonlocal property of shared state and violate of some inequality (CHSH, Mermin, Svetlichniy, etc.) using Bell theorem. For these, we use the full-quantum model of electro-optical phase modulation process including finite number of interacting modes [24]. This model asymptotically approaches the semi-classical one, which was considered in [23], when number of interacting modes goes to infinity. Testing a number of cases with different shared entangled states, we solve the optimization problem obtain corresponding modulation parameters, which prove state nonlocality for each case. Additionally, we estimate the region of detection settings, which reveals Bell nonlocality.

Our paper has the following structure. Section 2 is devoted to main definitions and mathematical model of multi-partite Bell test. In Section 3, we introduce the notion of frequency bin and derive multi-photon frequencyentangled quantum state, shared between all parties of quantum network. In Section 4, we describe generalized 
measurement procedure at each size of the network and formulate Svetlichny inequality for the case under investigation. Numerical modelling and solution of optimization problem for particular cases of entangled two (EPR) and three (GHZ and Wigner) states are in Section 5. Section 6 is devoted to discussion and conclude the paper.

\section{Modelling of multi-partite Bell test}

In a model of our theoretical Bell experiment, we will use notations following [1]. We set A (Alice), B (Bob), $\mathrm{C}$ (Charlie), ... as communicating parts of considered quantum network (see fig. 1). At each side of this network, there is a measurement device $D_{A}, D_{B}, D_{C}, \ldots$ with a definite input: $x^{(A)} \in\left\{x_{i}\right\}_{i=1}^{n_{A}}$ for $D_{A}, y^{(B)} \in\left\{y_{j}\right\}_{j=1}^{n_{B}}$ for $D_{B}, z^{(C)} \in\left\{z_{k}\right\}_{k=1}^{n_{C}}$ for $D_{C}, \ldots$. Possible outcomes of measurement procedure on each detector is denoted by $a \in\left\{a_{\alpha}\right\}_{\alpha=1}^{m_{A}}$ for Alice side, $b \in\left\{b_{\beta}\right\}_{\beta=1}^{m_{B}}$ for Bob side, $c \in\left\{c_{\gamma}\right\}_{\gamma=1}^{m_{C}}$ for Charlie, etc. The object of our interest will be a set $P$ of joint probabilities $p\left(a_{\alpha}, b_{\beta}, c_{\gamma} \ldots \mid x_{i}, y_{j}, z_{k} \ldots\right)$ to detect results $a_{\alpha}, b_{\beta}, c_{\gamma}, \ldots$ setting parameters $x_{i}, y_{j}, z_{k}, \ldots$ which usually called behaviour. For instance, local behavior $P_{L}$ of distributed detection system is supposed to satisfy the following condition for each join probability $p \in P_{L}$ :

$$
p\left(a_{\alpha}, b_{\beta}, c_{\gamma} \ldots \mid x_{i}, y_{j}, z_{k} \ldots\right)=\int_{\Lambda} d \lambda q(\lambda) p\left(a_{\alpha} \mid x_{i}, \lambda\right) p\left(b_{\beta} \mid y_{j}, \lambda\right) p\left(c_{\gamma} \mid z_{k}, \lambda\right) \ldots
$$

Here, $\lambda \in \Lambda$ is "hidden" variable taking value in space $\Lambda$ and distributed according to the probability density $q(\lambda)$. Quantum mechanics gives the following rule for calculating probabilities:

$$
p\left(a_{\alpha}, b_{\beta}, c_{\gamma} \ldots \mid x_{i}, y_{j}, z_{k} \ldots\right)=\operatorname{tr}\left(\rho_{A B C \ldots} M_{a_{\alpha} \mid x_{i}} \otimes M_{b_{\beta} \mid y_{j}} \otimes M_{c_{\gamma} \mid z_{k}} \otimes \ldots\right),
$$

that form quantum behaviour $P_{Q}$ of measurement apparatus. Here, $\rho_{A B C \ldots}$ is quantum state on common Hilbert space $H_{A} \otimes H_{B} \otimes H_{C} \otimes \ldots$, and $M$ are measurement operators (positive operator-valued measures) on corresponded subspace. Due to the fact that $P_{L} \subset P_{Q}$, there exist quantum states (nonlocal) with statistics different from that of correspondent to local behavior. This means that probability distribution of the form (1) for any quantum state $\rho_{A B C \ldots}$ does not exist. Inequality for detecting genuine multi-partite nonlocality was introduced [25] and generalized [26] by Svetlichny. It has the form:

$$
S_{N}=\left\langle S_{N-1} r_{N}^{\prime}\right\rangle+\left\langle S_{N-1}^{\prime} r_{N}\right\rangle \leq 2^{N-1},
$$

obtained from repeating the procedure which allowed us to build Svetlichny's inequality from CHSH:

$$
\begin{aligned}
S_{3}=\left\langle r_{1}^{\prime} r_{2} r_{3}\right\rangle+\left\langle r_{1} r_{2}^{\prime} r_{3}\right\rangle+\left\langle r_{1} r_{2} r_{3}^{\prime}\right\rangle-\left\langle r_{1}^{\prime} r_{2}^{\prime} r_{3}^{\prime}\right\rangle+\left\langle r_{1}^{\prime} r_{2}^{\prime} r_{3}\right\rangle+\left\langle r_{1}^{\prime} r_{2} r_{3}^{\prime}\right\rangle+\left\langle r_{1} r_{2}^{\prime} r_{3}^{\prime}\right\rangle & -\left\langle r_{1} r_{2} r_{3}\right\rangle \equiv \\
& \left\langle S r_{3}^{\prime}\right\rangle+\left\langle S^{\prime} r_{3}\right\rangle \leq 4
\end{aligned}
$$

Here it is supposed that all measurements are dichotomic and their results are denoted by $r_{j}= \pm 1$ and $r_{j}^{\prime}= \pm 1$, where $r_{1}=a, r_{2}=b, r_{3}=c, \ldots$.

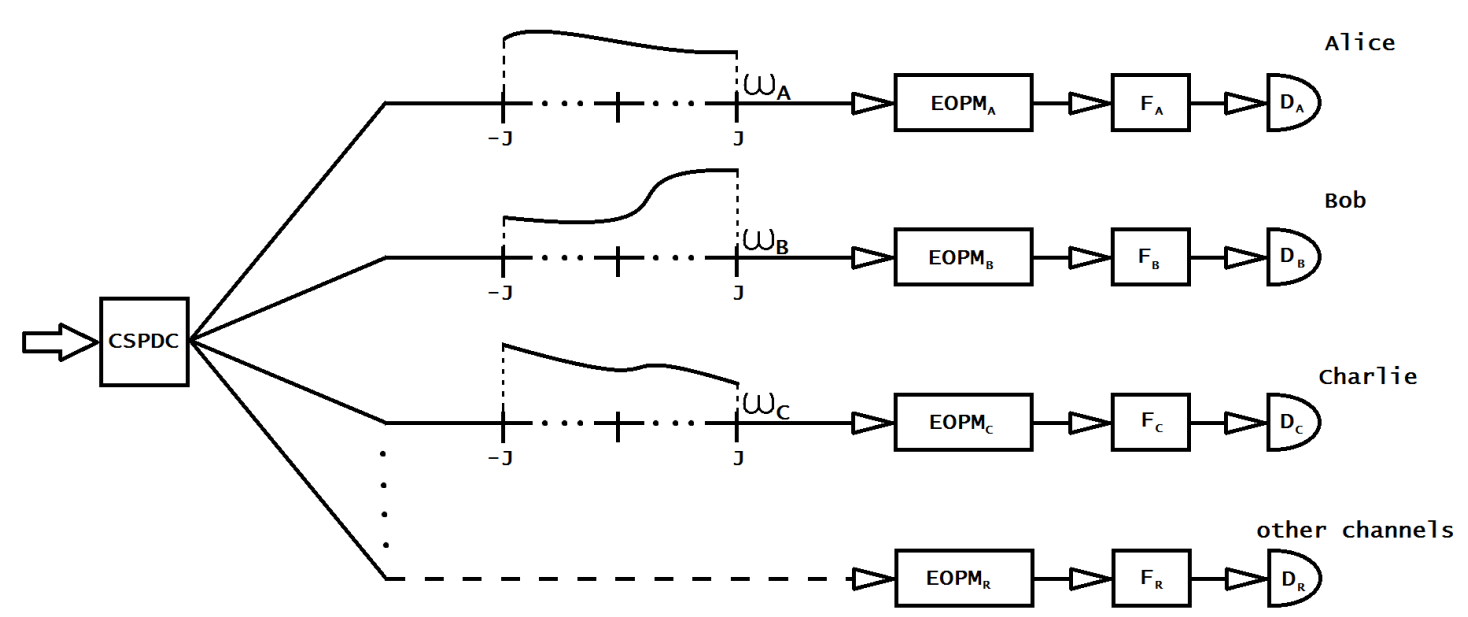

FIG. 1. Quantum network for $N$-photon experiment. The source is a pump laser that generates frequency-entangled photons resulting from cascade spontaneous parametric down-conversion process. In each channel, tuned EOPMs are located. Photons phases are modulated by EOPMs driven by signals at certain frequencies with respective pairs of parameters. $F_{R}$ for drop filters, $D_{R}$ for detectors (SSPDs) 
In the next section we will define common $N$-photon quantum multi-mode state shared between all $N$ parties of the quantum optical network and describe procedure revealing it's nonlocal properties using formalism presented in this section.

\section{Frequency-bin coding and multi-mode quantum states}

We will consider a process of entangled $N$-photon quantum state generation resulting, for example, from cascade spontaneous down conversion scheme. This process produce photons in frequency-entangled state of the form $\rho_{A B C \ldots}=|\chi\rangle\langle\chi|$, where:

$$
|\chi\rangle \equiv|\chi\rangle_{A B C \ldots}=\int_{-\infty}^{\infty} d \omega_{a} d \omega_{b} d \omega_{c} \ldots F\left(\omega_{a}, \omega_{b}, \omega_{c}, \ldots\right)\left|1_{\omega_{a}}\right\rangle_{A}\left|1_{\omega_{b}}\right\rangle_{B}\left|1_{\omega_{c}}\right\rangle_{C} \ldots
$$

Here, $F\left(\omega_{a}, \omega_{b}, \omega_{c}, \ldots\right)$ is spectral density distribution and $\left|1_{\omega_{r}}\right\rangle_{R}$ is denoted single photon state with frequency $\omega_{r}, r \in\{a, b, c, \ldots\}$ transmitted to channel $R \in\{A, B, C, \ldots\}$. Each channel $R$ is equipped with electro-optical phase modulator $\mathrm{EOPM}_{R}$, frequency filter $\mathrm{F}_{R}$ and photo-counter $\mathrm{D}_{R}$. Modulation frequency $\Omega_{M W}$ for all EOPM $R$ is assumed to be the same, while other parameters may be different. The action of EOPMs on arriving state can only change the frequencies by integer multiples of $\Omega_{M W}$. For the next, following [21] we introduce the notion of frequency bin: all photons whose frequencies are contained in an interval $\left[\omega_{0}-\Omega / 2, \omega_{0}+\Omega / 2\right]$ are grouped into a single frequency bin centred on frequency $\omega_{0}$. Assuming $\Omega \ll \Omega_{M W}$ we can write our initial state in the discretized form:

$$
|\chi\rangle_{A B C \ldots}=\sum_{a, b, c, \ldots \in Z} F_{a, b, c, \ldots}\left|1_{a}\right\rangle_{A}\left|1_{b}\right\rangle_{B}\left|1_{c}\right\rangle_{C} \ldots
$$

where we introduced $\omega_{r} \equiv \omega_{0}+r \Omega_{M W}$ and dropped leaving only index $r$. Considering photon creation operator $a_{r, R}^{\dagger}$ on frequency $\omega_{r}$ in channel $R$ and fixing order for $R$ (to drop $R$ ), we finally obtain:

$$
|\chi\rangle_{A B C \ldots}=\sum_{a, b, c, \ldots \in Z} F_{a, b, c, \ldots} a_{a}^{\dagger} a_{b}^{\dagger} a_{c}^{\dagger} \ldots|0\rangle .
$$

The state of this form will be the subject of our investigation of it's nonlocal properties with the help of inequalities (3) and (4).

\section{Detection procedure and inequality derivation}

As it was mentioned above, each side of our quantum network is equipped with EOPM, filter and photocounter. To realize the procedure of state nonlocality detection using inequality (3) we consider these three devices as generalized measurement apparatus, whose input is parametrized by modulator parameters $(\varphi, \tilde{\alpha}, \tilde{\beta})$ and filter properties. The next question is: what agreement should reach all parties about the inputs and outputs of their measurement devices to reveal nonlocal properties of state (7). We restrict ourselves by two sets of modulation parameters as to different inputs for each generalized detector. And our goal now is to obtain optimal values of these parameters to maximize functional (3) solving corresponding optimization problem. To do this, we consider full quantum model of EOPM, investigated in [24] and write the modulator action as:

$$
U|\chi\rangle_{A B C \ldots}=\sum_{a^{\prime}, b^{\prime}, c^{\prime}, \ldots \in Z} F_{a^{\prime}, b^{\prime}, c^{\prime}, \ldots} \tilde{a}_{a^{\prime}}^{\dagger} \tilde{a}_{b^{\prime}}^{\dagger} \tilde{a}_{c^{\prime}}^{\dagger} \ldots|0\rangle,
$$

where evolution operator $U \equiv U(\varphi, \tilde{\alpha}, \tilde{\beta})$ and output operators $\tilde{a}_{r^{\prime}}^{\dagger}$ are expressed through input ones with use of the formula:

$$
\tilde{a}_{r^{\prime}}^{\dagger}=U^{\dagger} a_{r}^{\dagger} U=e^{-i\left(\omega_{0}+r^{\prime} \Omega_{M W}\right) T} \sum_{r=-J}^{J}(-1)^{r} e^{-i\left(r+r^{\prime}\right) \tilde{\alpha}} d_{r^{\prime} r}^{J}(\tilde{\beta}) e^{i\left(r^{\prime}-r\right) \varphi} a_{r}^{\dagger} \equiv \sum_{r=-J}^{J} M_{r^{\prime} r}^{(J)} a_{r}^{\dagger},
$$

where $T$ is modulation time, $(2 J+1)$ is number of interacted modes in modulator and $d_{\mu \nu}^{J}(\tilde{\beta})$ is the Wigner d-function. Using (9), Eq. (8) may be written as:

and

$$
U|\chi\rangle_{A B C \ldots}=\sum_{a, b, c, \ldots \in Z} \tilde{F}_{a, b, c, \ldots} a_{a}^{\dagger} a_{b}^{\dagger} a_{c}^{\dagger} \ldots|0\rangle
$$

$$
\tilde{F}_{a, b, c, \ldots}=\sum_{a^{\prime}, b^{\prime}, c^{\prime}, \ldots \in Z} F_{a^{\prime}, b^{\prime}, c^{\prime}, \ldots} M_{a^{\prime} a}^{(J)} M_{b^{\prime} b}^{(J)} M_{c^{\prime} c}^{(J)} \ldots
$$


For physical reasons and from the fact that evolution operator $U$ is unitary, we have $\|F\|_{2}=\|\tilde{F}\|_{2}=1$, where $\|F\|_{2}=\sum_{a, b, c, \ldots \in Z}\left|F_{a, b, c, \ldots}\right|^{2}$.

After determination of two inputs $\left(\tilde{\alpha}_{i}, \tilde{\beta}_{i}\right)_{R}, i=1,2$ for each measurement device, we characterize measurement result in channel $R$ by random variable $\theta_{R}= \pm 1$, that takes value $\theta_{r, R}=1$ then there was a photo-count at bin $\omega_{r}$ in channel $R$ and $\theta_{R}=-1$ otherwise. Joint probability for the events when all photons are detected at frequency $\omega_{a_{\alpha}}$ in channel $A, \omega_{b_{\beta}}$ in channel $B, \omega_{c_{\gamma}}$ in channel $C, \ldots$ under input parameters $x_{i}=\left(\tilde{\alpha}_{i}, \tilde{\beta}_{i}\right)_{A}$, $y_{j}=\left(\tilde{\alpha_{j}}, \tilde{\beta}_{j}\right)_{B}, z_{k}=\left(\tilde{\alpha_{k}}, \tilde{\beta_{k}}\right)_{C}$, etc. is

$$
p\left(a_{\alpha}, b_{\beta}, c_{\gamma}, \ldots \mid x_{i}, y_{j}, z_{k}, \ldots\right)=\left|\left\langle 1_{a_{\alpha}} 1_{b_{\beta}} 1_{c_{\gamma}}|U| \chi\right\rangle_{A B C \ldots}\right|^{2}=\left|\tilde{F}_{a_{\alpha} b_{\beta} c_{\gamma} \ldots}\right|^{2} .
$$

To estimate the "true" statistics of initial state before modulation process, we use Bayes' theorem, which gives conditional probability:

$$
p_{B}\left(a, b, c, \ldots \mid a^{\prime}, b^{\prime}, c^{\prime}, \ldots\right)=\frac{\left|F_{a^{\prime}, b^{\prime}, c^{\prime}, \ldots} M_{a^{\prime} a}^{(J)} M_{b^{\prime} b}^{(J)} M_{c^{\prime} c}^{(J)} \ldots\right|^{2}}{\sum_{a^{\prime}, b^{\prime}, c^{\prime}, \ldots \in Z}\left|F_{a^{\prime}, b^{\prime}, c^{\prime}, \ldots} M_{a^{\prime} a}^{(J)} M_{b^{\prime} b}^{(J)} M_{c^{\prime} c}^{(J)} \ldots\right|^{2}} .
$$

Taking into account the possible values of random variables $\theta_{r, R} \in\{ \pm 1\}$, we can write:

$$
\left\langle\theta_{r_{1}, A}, \theta_{r_{2}, B}, \theta_{r_{3}, C}, \ldots\right\rangle \equiv\left\langle\theta_{a, A}, \theta_{b, B}, \theta_{c, C}, \ldots\right\rangle=\operatorname{sgn}\left(\theta_{a, A} \theta_{b, B} \theta_{c, C} \ldots\right) p_{B}\left(a, b, c, \ldots \mid a^{\prime}, b^{\prime}, c^{\prime}, \ldots\right) .
$$

Thus, we arrive to Svetlichny inequality for frequency-encoded multi-mode quantum states. The next section will be devoted to optimization problem for modulator parameters which maximize Bell functional and reveal nonlocality of shared multi-photon entangled quantum state.

\section{Numerical modelling: bipartite and tripartite cases}

In this section, we will represent the results of some numerical analysis.

Let us choose the system to have 6 interacted modes (this means $J=2.5$ ) in every channel. Photons in every channel could have their own spectrum, but without loss of generality let us make them all to be equal. We have there only 6 frequencies from $\omega_{0}-2.5 \cdot \Omega_{M W}$ to $\omega_{0}+2.5 \cdot \Omega_{M W}$.

\subsection{Bipartite case}

In the section, we investigate two-particle case in a specific scheme, which approve Bell results and in particular CH74 (Clauser-Horne, [7]) inequality in a frequency domain. This setup was initially proposed in [21] to test CH74 inequality using phase modulated photons generated in spontaneous down conversion (SPDC) process. Using the approach presented above, we now can determine $S$ in $\mathrm{CH} 74$ inequality and formulate optimization problem, which has a solution. We got the maximum value for $S$ of 2.389. This result corresponds with [21], but in our approach we can operate with any frequency spectrum. We show plots in order to get understanding of nonlocality domain pattern in Fig. 2.

\subsection{Tripartite case}

Here, we are going to modelling a priory entangled tripartite states in order to verify whether they are nonlocal.

5.2.1. Wigner-state. We start with Wigner state expressed in terms of frequency entangled photon triplet:

$$
|W\rangle=\left|-0.5_{A}, 1.5_{B}, 2.5_{C}\right\rangle+\left|-0.5_{A}, 2.5_{B},-1.5_{c}\right\rangle+\left|2.5_{A},-0.5_{B},-1.5_{C}\right\rangle,
$$

where i.e. $|-0.5\rangle$ means $\left|\omega_{0}-0.5 \dot{\Omega}_{R F}\right\rangle$ and $A, B, C$ represent Alice's, Bob's and Charlie's channels respectively.

We show the spectrum probability density of the state before modulation in the Fig. 3(a).

Every channel has its own EOM [24]. In Fig. 4(a) the spectrum probability density of the Wigner state after modulation is shown.

For this state we build Svetlichy inequality $S\left(\alpha_{11}, \beta_{11}, \alpha_{21}, \beta_{21}, \alpha_{31}, \beta_{31}, \alpha_{12}, \beta_{12}, \alpha_{22}, \beta_{22}, \alpha_{32}, \beta_{32}\right)$. We found the maximum of the functional in this case as 4.914 at point

$$
\{1.946,4.444,5.094,3.691,6.161,0.445,2.371,5.121,0.094,5.482,3.799,6.224\} .
$$

Now, we can describe domain of nonlocality for our state varying any parameters in 18 . For clarity we vary the second EOM parameter of first channel $\beta_{11}$ and the same second EOM parameter of the second channel $\beta_{21}$. 


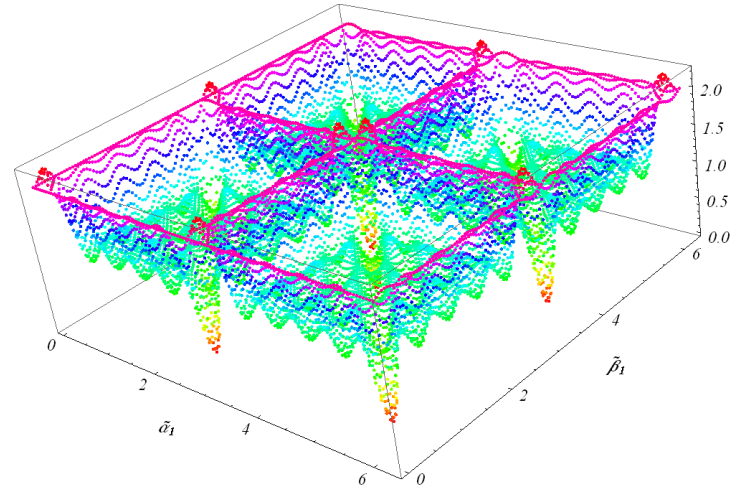

(a)

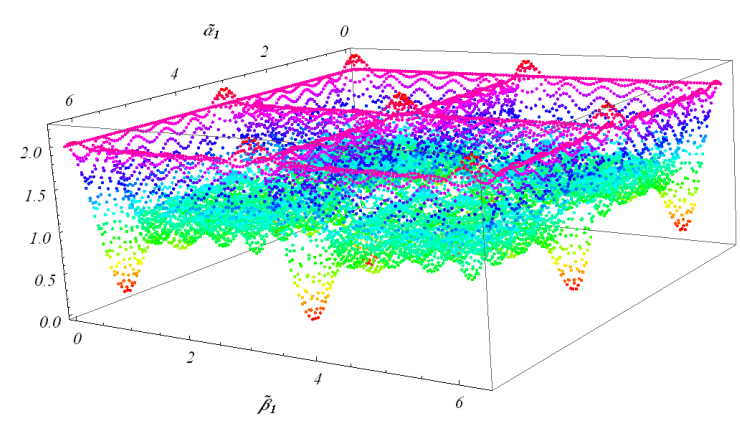

(b)

FIG. 2. We build 3d-surface of Bell functional, varying two of the parameters. All the parameters are in $[0 ; 2 \pi]$. The surface is projection the Bell inequality onto pair of parameters $\tilde{\alpha}_{1}$ and $\tilde{\beta}_{1}$ given fixed other parameters $\left(S\left(0,0,0,0,0, \tilde{\alpha_{1}}, 0.3, \tilde{\beta_{1}}\right)\right)$. There are extremes at points of $(x ; y)$ where $x, y \in 0, \pi, 2 \pi$. (a) and (b) displays $S$

$$
f(n, m, l=2.5)
$$
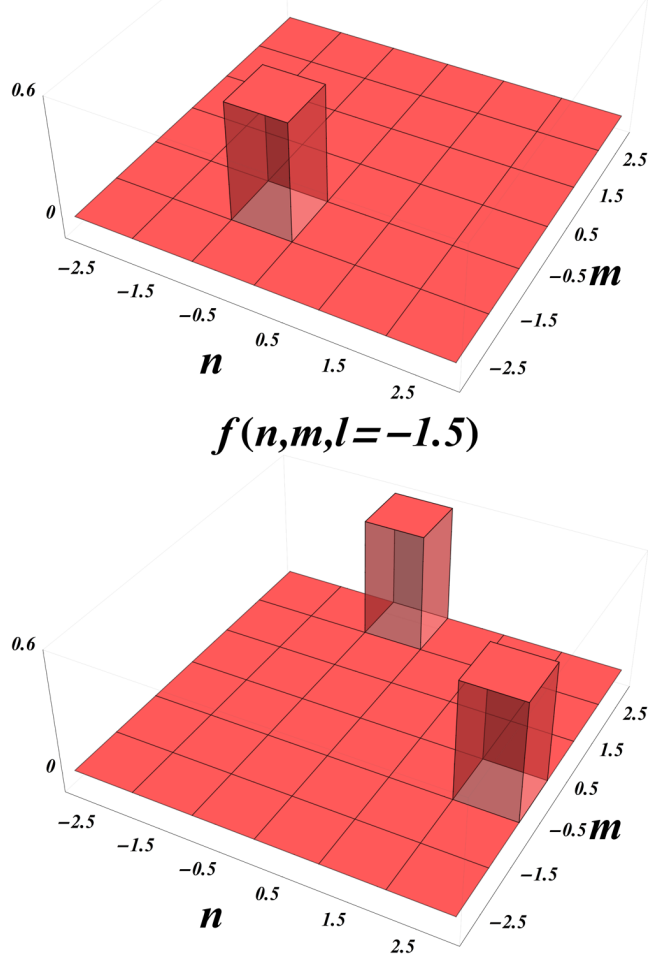

(a) $f(n, m, l=1.5)$
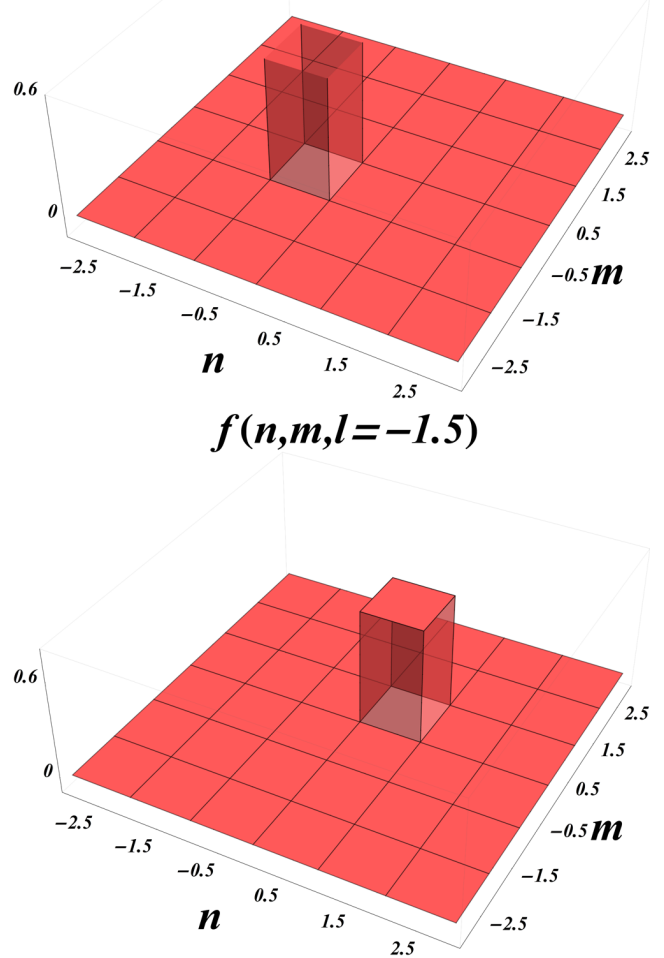

(b)

FIG. 3. (a) The spectrum probability density of the Wigner state before modulation for certain third frequency $l$. $m$ denotes the first channel frequency, $n$ denotes the second. For other $l$ the probability function is equal to zero. (b) The spectrum probability density of the GHZ state before modulation for certain third frequency $l$. As before for other $l$ the probability function is equals to zero 


\section{$f(n, m)$}

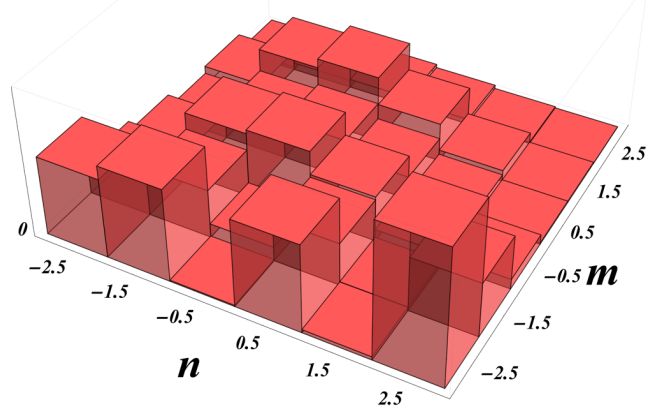

(a) $f(n, m)$

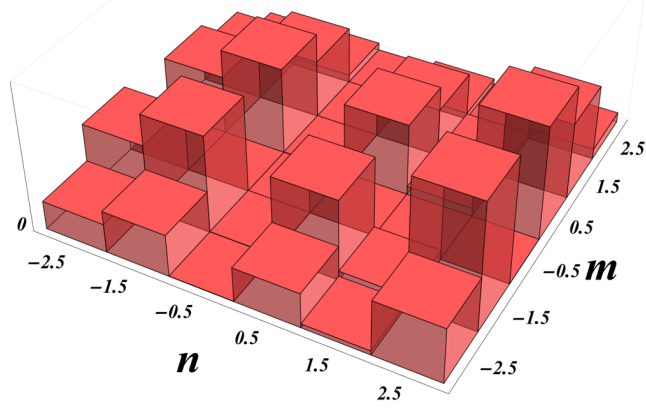

(b)

FIG. 4. (a) The spectrum probability density of the Wigner state after modulation for any third frequency $l$. $m$ denotes the first channel frequency, $n$ denotes the second. (b) The spectrum probability density of the GHZ state after modulation for any third frequency $l . m$ denotes the first channel frequency, $n$ denotes the second

Domains violating the Svetlichny inequality are shown in Fig.5(a) (as was said before the local boundary of the inequality is 4 ).

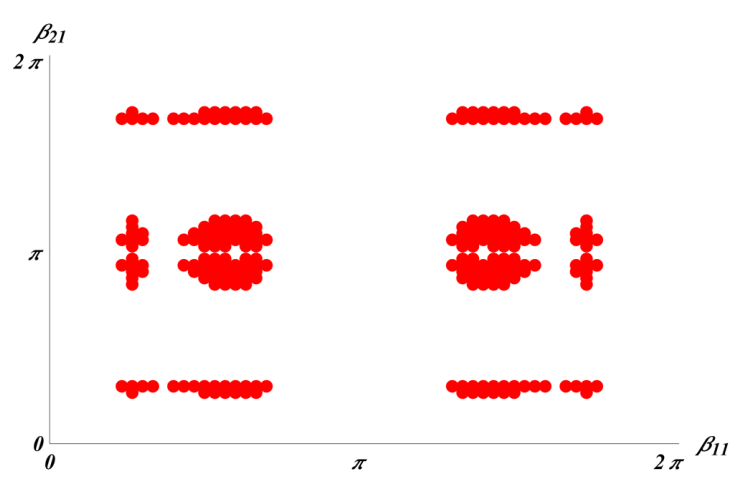

(a)

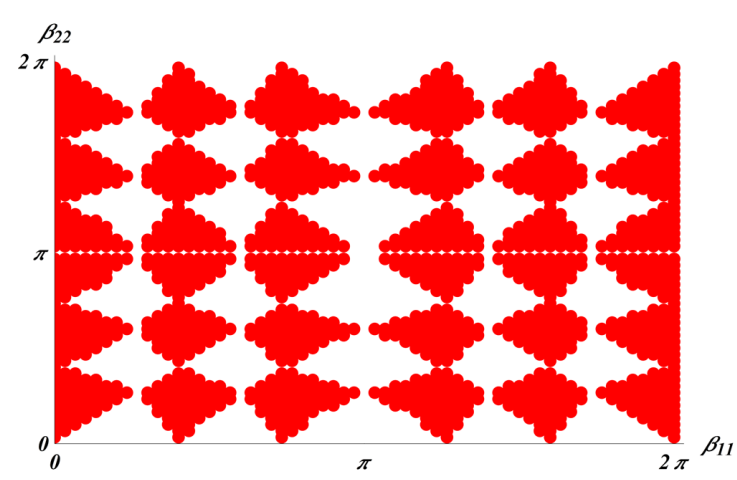

(b)

FIG. 5. (a) The nonlocal domains of Wigner state in 2d-projection. (b) The nonlocal domains of GHZ state in 2d-projection

5.2.2. GHZ-state. We designed the method that allows us to check any state for nonlocality. For this purpose, we need to have only spectrum probability density for a state. We show this with the example of another entangled state, such as GHZ-state. The spectrum density of GHZ-state is

$$
|G H Z\rangle=\left|-0.5_{A},-0.5_{B}, 1.5_{C}\right\rangle+\left|0.5_{A}, 0.5_{B},-1.5_{C}\right\rangle
$$

and is shown before modulation in Fig.3(b) and after modulation in Fig. 4(b).

For the current state, we obtained a violation 4.994 at the point

$$
\{4.61,1.25,3.095,3.948,0.055,6.213,0.69,5.499,4.129,0.517,4.787,2.15\} \text {. }
$$

Now we vary $\beta_{11}$ and $\beta_{22}$ in order to get nonlocality domain of GHZ state in Fig.5(b).

\section{Conclusion}

The current work is devoted to Bell inequalities in multi-partite systems. We presented Bell formalism applicable to our problem and formulated CHSH inequality in case of N-dimension problem. Following [21] we formulate common state which could be modulated in our approach. Also, we derive joint probabilities to construct genuine spectral density distribution. Using the approach presented in [24], we build the method which 
allows us to test any state of nonlocality and show the operation of this method on different states. We checked a priori entangled bipartite state and find the point violated $\mathrm{CH} 74$ inequality $S$ maximally. The result corresponding with [21]. We presented violating of $W$ and $G H Z$ states and obtained domains of nonlocality in all. We can generalize the approach to any-dimensional quantum nanosystem system for any entangled or not entangled state.

\section{Acknowledgements}

This work was partially financially supported by the Government of the Russian Federation (grant 08-08), by grant 16-11-10330 of Russian Science Foundation.

\section{References}

[1] Bruner N., et al. Bell nonlocality. Review of Modern Physics, 2014, 86 (2), P. 419-478.

[2] Horodecki R. Quantum entanglement. Reviews of Modern Physics, 2009, 81 (2), P. 865-942.

[3] Gisin N. Bell's inequality holds for all non-product states. Physics Letters A, 1991, 154 (5-6), P. 201-202.

[4] Barret J. Nonsequential POVM's on entangled mixed states do not always violate a Bell inequality. Physical Review A, 2002 , 65 (4), 042302.

[5] Masanes L., Liang Y.-C., Doherty A.C. All Bipartite Entangled States Display Some Hidden Nonlocality. Physical Review Letters, 2008, 100 (9), 090403.

[6] Bell J.S. On the Einstein Podolsky Rosen Paradox. Physics, 1964, 1 (3), P. 195-200.

[7] Clauser J.F., Horne M.A. Experimental consequences of objective local theories. Phys. Rev. D, 1974,10, P. 526.

[8] Clauser J.F., Horne M.A., Shimony A., Holt R.A. On the Einstein Podolsky Rosen Paradox. Physical Review Letters, 1969, 23 (15), P. 880 .

[9] Mermin N.D. Extreme quantum entanglement in a superposition of macroscopically distinct states. Physical Review Letters, 1990, 65 (15), P. 1838 .

[10] Belinskii A., Klyshko D. Interference of light and Bell's theorem. Physics-Uspekhi, 1993, 36 (8), P. 653.

[11] Lodahl P., Mahmoodian S., Stobbe S. Interfacing single photons and single quantum dots with photonic nanostructures. Reviews of Modern Physics, 2015, 87 (2), P. 347.

[12] Gisin N., et al. Quantum cryptography. Reviews of Modern Physics, 2002, 74 (1), P. 145-195.

[13] Soorat R., Madhuri K., Vudayagiri A. Random number generator for cryptography. Nanosystems: Physics, Chemistry, Mathematics, 2017, 8 (5), P. 600-605.

[14] Ivanova A.E., Chivilikhin S.A., Gleim A.V. The use of beam and fiber splitters in quantum random number generators based on vacuum fluctuations. Nanosystems: Physics, Chemistry, Mathematics, 2016, 7 (2), P. 378-383.

[15] Kues M., et al. On-chip generation of high-dimensional entangled quantum states and their coherent control. Nature, 2017 , 546, P. 622.

[16] Zeilinger A., et al. Significant-loophole-free test of Bell's theorem with entangled photons. Physical Review Letters, 2015, 115 (25), 250401.

[17] Knill E., et al. Strong loophole-free test of local realism. Physical Review Letters, 2015, 115 (25), 250402.

[18] Capmany J., Fernandez-Pousa C.R. Realization of Single-Photon Frequency-Domain Qubit Channels Using Phase Modulators. IEEE Photonics Journal, 2012, 4 (6), P. 2074-2084.

[19] Lukens J.M., Lougovsky P. Frequency-encoded photonic qubits for scalable quantum information processing. Optica, 2017,4 (1), 010008.

[20] Lu H.-H., et al. Electro-Optic Frequency Beam Splitters and Tritters for High-Fidelity Photonic Quantum Information Processing. Physical Review Letters, 2018, 120 (3), 030502(6).

[21] Olislager L., et al. Implementing two-photon interference in the frequency domain with electro-optic phase modulators. New Journal of Physics, 2012, 14, 043015.

[22] Guo X., Mey Y., Du S. Testing the Bell inequality on frequency-bin entangled photon pairs using time-resolved detection. Optica, 2017, 4 (4), 040388.

[23] Capmany J., Fernandez-Pousa C.R. Quantum modelling of electro-optic modulators. Laser Photonics Review, 2011,5 (6), P. 750-772.

[24] Miroshnichenko G.P., et al. Algebraic approach to electro-optic modulation of light: exactly solvable multimode quantum model. Journal of the Optical Society of America B, 2017, 34 (6), 061177.

[25] Svetlichny G. Distinguishing three-body from two-body nonseparability by a Bell-type inequality. Physical Review D, 1987,35 (10), 3066.

[26] Seevinck M., Svetlichny G. Bell-Type Inequalities for Partial Separability in N-Particle Systems and Quantum Mechanical Violations. Physical Review Letters, 2002, 89 (6), 060401. 